\title{
Assessment of interregional economic integration based on the integral indicator of the level of socio-economic development
}

\author{
Liudmila Borisova ${ }^{1}$ and Dina Borisova ${ }^{1 *}$ \\ ${ }^{1}$ Don State Technical University, sq. Gagarina, 1, Rostov-on-Don, 344010, Russia
}

\begin{abstract}
To search for and identify rational and effective solutions on deepening interregional cooperation, an unbiased assessment of the existing level, potential, and restrictions of the process of interregional integration is required. For assessing the state and prospects of development of interregional economic integration (along with other its types: production and technological, information and communication etc.), the analysis of the state and development trends of interregional commodity exchange as a carrier of integrational and economic functions is preferential. Methods and tools of investigating the processes of interregional economic integration, analysis and assessment of its existent level, used for these purposes in practice, do not always adequately reflect real conditions of functioning of economically integrated regions. Therefore, the need for developing well-known and working-out new approaches and methods for investigating the processes of interregional integration, assessment of tools and methods of managerial decision making, in order to enhance it and improve efficiency, prove the relevance of the chosen subject of investigation. To assess the degree of interregional interaction of the southern regions of Russia, a proprietary methodology is proposed.
\end{abstract}

\section{Introduction}

Economic interests of the entities of transregional ties act as a system-forming basis of this process. The problems of mathematical and cognitive modeling, including complex socioeconomic systems, are considered in the papers of N. Abramova, Z. Avdeeva, T. Baranovskaya, Y. Bershitsky, A. Burda, G. Gorelova, I. Katsko, V. Kulba, V. Maksimov, R. Atkin, J. Casti, E. Popova, F. Roberts, A. Trubilin, V. Loiko, E. Lutsenko, V. Khoroshevsky at al [1-4]. The authors did not consider the possibility of cognitive modeling taking into account fuzziness of initial data and multicriteria of the problem. The analysis has shown that despite a wide range of choice, the issues of assessing the current level and substantiating the development directions of the process of interregional economic integration remain insufficiently developed.

\footnotetext{
*Corresponding author: borisovalv09@mail.ru
} 
In this regardworking out the mechanisms for assessing instrumental and functional potential of interregional integration as a driver of socio-economic development of partner regions for its improvement makes it possible to implement modeling and quantitative assessment of the subject area under consideration.

A comparative analysis of the existing methods for assessing the level of a region development made it possible to single out such problems as: monitoring the degree of development of socio-economic systems (SES), stratification of regions and their ranking, extrapolation of indicator values of development of regional SES.

In contemporary conditions, various methods are applied for assessing the state of socio-economic development of the region, as well as different kinds of ratings [5-9].

The use of rating techniques incorporates the following stages:

-selection of initial socio-economic indicators;

- creating a system for comparing indicators;

- construction of summary ratings;

- ranking the regions according to ratings.

Despite the distribution of rating assessments, in practice it is expedient to use both index and criterion methods.

The problem of assessing a socio-economic level of development of regions is multicriteria, and to solve this problem it is necessary to take into account a lot of heteronymous and multidirectional indicators (GRP, average per capita income of the population, volume of retail trade, level of unemployment, rate of employment in the economy, fixed assets value, crime rate etc.). The application of the multicriteria method at the initial stage of investigation presupposes the formation of a complex of input factors of socio-economic development of partner regions for building a generalized indicator (integral indicator of the development level).

This generalized indicator, presented in the form of a super-criterion provides for the transition of a multicriteria problem to a single-criterion one. In practice, this transition means the formation of a super-criterion of the form:

$$
Q(x)=Q\left(q_{1}(x), q_{2}(x), \ldots q_{N}(x)\right)
$$

This approach makes it possible to order the solution options on the basis of $Q$ values, and based on the ordering to determine the best variant. It is also necessary to determine NAP (direction of interaction) - a sign of the influence of the factor on the resulting sign. The NAP indicator can take values 1 or 0 depending on the influence of a factor on the resulting sign (respectively direct or reverse).

One of the most effective ways of constructing a super criterion, when performing a multicriteria analysis, is the generalized Harrington desirability function. This function allows us to represent the natural values of particular criteria on a dimensionless desirability scale which varies from 0 to 1 . The values correspond to some points on the curve which is given by the equation:

$$
\mathrm{d}=\mathrm{e}^{-\mathrm{e}^{4-\mathrm{x}}}
$$

Variation range of values $d$ from 0 to 1 . It should be noted, that in the middle zone of the definition area, the function sensitivity is much larger than in the extreme points ( 0 and 1). To reconcile the actual values of $x$ with the numerical values of the scale, a scaling factor $\mathrm{M}$ is introduced: 


$$
M_{i}=\frac{x_{i \max }-x_{i \min }}{X_{B}-X_{A}}
$$

To convert the real values of factors into dimensionless ones, the following formulae are used:

$$
x_{i}=X_{A}+\frac{x_{i}^{p}-x_{i m i n}}{M_{i}} X_{i}=X_{B}+\frac{x_{i}^{p}-x_{i m i n}}{M_{i}}
$$

Respectively for NAP $=1$ and $\mathrm{NAP}=0$.

Here $X_{A}, X_{B}$ are the values of functions at the inflexion points; $x_{i}$ is a code value of the factor; $x_{i}^{p}$ is a natural value of the factor.

Next, the particular values of the desirability function $d_{i}$. The value of the super criterion (generalized indicator D) is determined as a geometrical mean value $d_{i}$ :

$$
D=\sqrt[n]{\prod_{i=1}^{n} d_{i}}
$$

The generalized desirability function $\mathrm{D}$ is some abstract construction and possesses the following qualities: the function is quantitative, specific, general, and universal indicator of the object quality (along with the properties of adequacy, efficiency, and statistical sensitivity). In this regard, function $\mathrm{D}$ can be used as a criterion for assessing the level of socio-economic development of regions.

The results of assessing the level of interregional integration of the processes imply the strength of interaction between partner regions [10-11].

Thus, for a comparative assessment of regional development in the Southern Federal District, we have proposed an integral indicator of the socio-economic development of the region. This indicator includes, indirectly, both the social component of the process, and the transport and logistics potential of the region. That is, it takes into account a lot of factors that affect the level of interregional economic integration, such as the «level of transport communication» and «the level of communications development», which incorporate groups of private subcriteria.

\section{Methods}

The performed earlier analysis of existing methods and approaches to the investigation of assessment of the development level of regional partners made it possible to develop a methodology of constructing an integral indicator of socio-economic development.

The methods for assessing the level of interregional economic integration on the basis of integral indicator allows us to take into consideration transport and logistics subsystems of partner regions of the Southern Federal District as an input factor "level of development of transport communication" (LTC) cargo turnover, million tons; density of paved roads per $1000 \mathrm{~km}^{2}$ etc. [12].

The calculation of the integral indicator also makes it possible to take into account the state of the level of development of communication systems between partner regions in the South of Russia as an input factor «the level of communication development» (LCD).

The formalized representation of the studied input factors: SED, LTC, LCD, for the most part, characterized by quality uncertainty, as well as the indicator of commodity turnover (CT) of the partner regions of the Southern Federal District, it is reasonable to implement on the basis of the apparatus of fuzzy logics. The group of indicators of 
commodity turnover includes indicators characterizing the import/export of products, as well as the volume of commodity turnover for three consolidated types of products: food products (FP); non-food products (NFP); industrial and technical products (ITP) with the regions of the Southern Federal District.

The proposed indicator, based on the Harrington desirability function, allowed us to give an adequate comparative assessment of the development levels of the regions of the Southern Federal District, providing for a great number of indicators, describing the Southern Federal District. Fig. 1 presents the generalized diagram of constructing an integral indicator of efficiency.

The methodology for assessing the efficiency of interregional economic integration as a tool for the economic growth of partner regions is a new approach based on the calculation of the integral indicator of efficiency of socio-economic development of these regions. The application of the criterion approach allows us to consider together both heteronymic and competing factors: «socio-economic indicators (SEI), commodity turnover (CT), the level of communications development (LCD), and the level of transport communication (LTC) with its infrastructure, incorporating transport corridors, intermodal hubs etc.».

Therefore, when analyzing the participation of these factors in the integrational processes, it seems appropriate to take into account their availability, and also the form of promotion to the development of integration processes [13-15].

\section{Results}

The application of the proposed methodology for determining the integral indicator of the level of socio-economic development (Fig.1) makes it possible to transform the problem with many criteria and consider it as a single-criterion for assessing the level of development of a particular region taking into account multidirectional and multidimensional socio-economic factors.

The main purpose of the study of interregional interaction is to identify trends in the socio-economic development of the entities of the federal district with consideration to the main influencing factors.

A quantitative assessment of the level of interregional integration of the RR with the regions of the Southern Federal District will provide an opportunity to develop corrective measures to intensify the integration processes based on factors that take into account the qualitative and quantitative nature of the information base. We calculate the integral indicator of the development level of the Southern Federal District at runtime for 2018 (Table 1-3).

The calculations show that the level of development of SED tends to grow in all the entities of the Southern Federal District, and the first place belongs to the Krasnodar Territory. In the socio-economic development of the entities of the district, among the main indicators we can distinguish:

1) increasing the level of entrepreneurial and innovative activity;

2) improving the demographic situation and the quality of life of the population;

3) overcoming the shortage of qualified personnel;

4) reducing the degree of depreciation of fixed assets;

5) reducing the deficit of the consolidated budget of the region and its high subsidization;

6) legitimization of the "hidden" (shadow) economy (informal sector). 


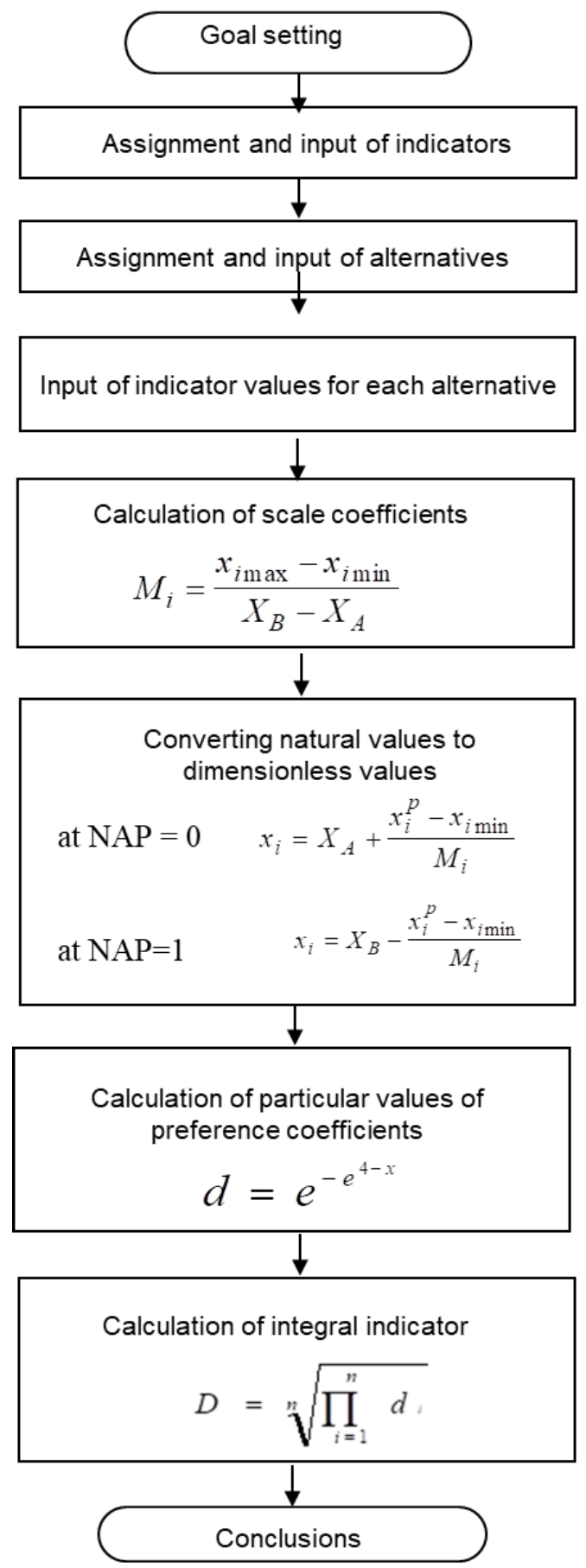

Fig. 1. Generalized diagram for constructing an integral indicator of socio-economic development of regions. 
Table 1. The value of development indicators for the regions of the Southern Federal District for 2018.

\begin{tabular}{|c|l|c|c|c|c|}
\hline \multirow{2}{*}{$\begin{array}{c}\text { № } \\
\text { Indi } \\
\text { cator }\end{array}$} & \multicolumn{1}{|c|}{ Nameoftheindicator } & \multicolumn{3}{|c|}{ Sourcedata } \\
\cline { 3 - 6 } & NAP & $\min$ & $\max$ & Mi \\
\hline 1 & GRP, billion rubles (или RUB bn) & + & 30 & 5000 & 2523 \\
\hline 2 & $\begin{array}{l}\text { Volume of investments in fixed assets, billion } \\
\text { rubles }\end{array}$ & + & 10 & 1000 & 503 \\
\hline 3 & Volume of retail trade, billion rubles & + & 10 & 2000 & 1010 \\
\hline 4 & $\begin{array}{l}\text { Average per capita income of the population, } \\
\text { thousand rubles/year }\end{array}$ & + & 130 & 500 & 188 \\
\hline 5 & Crimes per 100 thousand people of the population & - & 500 & 2000 & 761 \\
\hline 6 & Birth rate per 1000 people & + & 5 & 20 & 8 \\
\hline 7 & Death rate per 1000 & - & 5 & 20 & 8 \\
\hline 8 & Morbidity rate per 1000 people & - & 500 & 1000 & 254 \\
\hline 9 & Shipped goods of local production, billion rubles & + & 10 & 3000 & 1518 \\
\hline 10 & Unemployed, thousand people & - & 10 & 3000 & 1518 \\
\hline 11 & Employed in the economy, thousand people & + & 100 & 3000 & 1472 \\
\hline 12 & Cost of fixed assets, billion rubles & + & 100 & 6000 & 2995 \\
\hline
\end{tabular}

Table 2. The value of development indicators for the regions of the Rostov region, Krasnodar Territory, Volgograd region for 2018.

\begin{tabular}{|c|c|c|c|c|c|c|c|c|c|}
\hline \multirow{2}{*}{$\begin{array}{c}\text { № } \\
\text { indicator }\end{array}$} & \multicolumn{3}{|c|}{ Rostovregion } & \multicolumn{3}{c|}{ KrasnodarTerritory } & \multicolumn{3}{c|}{ Volgograd region } \\
\cline { 2 - 11 } & Meaning & $\mathrm{Xi}$ & $\mathrm{di}$ & Meaning & $\mathrm{Xi}$ & $\mathrm{di}$ & Meaning & $\mathrm{Xi}$ & $\mathrm{di}$ \\
\hline 1 & 1347 & 4.0 & 0.39 & 2225 & 4.4 & 0.51 & 771.4 & 3.8 & 0.30 \\
\hline 2 & 252 & 4.0 & 0.37 & 484.1 & 4.4 & 0.54 & 184.4 & 3.8 & 0.32 \\
\hline 3 & 920 & 4.4 & 0.52 & 1368 & 4.8 & 0.66 & 389.6 & 3.9 & 0.33 \\
\hline 4 & 349 & 4.6 & 0.61 & 412.4 & 5.0 & 0.70 & 273.7 & 4.2 & 0.47 \\
\hline 5 & 1314 & 4.4 & 0.52 & 1216 & 4.5 & 0.56 & 1529 & 4.1 & 0.42 \\
\hline 6 & 9 & 4.0 & 0.39 & 11.5 & 4.3 & 0.51 & 9.4 & 4.1 & 0.41 \\
\hline 7 & 13.3 & 4.4 & 0.51 & 12 & 4.5 & 0.57 & 13.3 & 4.4 & 0.51 \\
\hline 8 & 893 & 3.9 & 0.35 & 688 & 4.7 & 0.63 & 712.1 & 4.6 & 0.60 \\
\hline 9 & 1356 & 4.4 & 0.52 & 2754 & 5.3 & 0.77 & 1125.3 & 4.2 & 0.46 \\
\hline 10 & 167 & 5.3 & 0.78 & 143 & 5.4 & 0.78 & 72.6 & 5.4 & 0.79 \\
\hline 11 & 1935 & 4.7 & 0.63 & 2671 & 5.2 & 0.76 & 1226 & 4.2 & 0.47 \\
\hline 12 & 3049 & 4.5 & 0.55 & 6260 & 5.5 & 0.82 & 2326 & 4.2 & 0.47 \\
\hline $\begin{array}{l}\text { The value of } \\
\text { the integral } \\
\text { indicatorof } \\
\text { the level of } \\
\text { development }\end{array}$ & & & & & & & & & \\
\hline
\end{tabular}


Table 3. The value of development indicators for the regions of the Astrakhan region, Republic of Adygea, Republic of Kalmykia for 2018.

\begin{tabular}{|c|c|c|c|c|c|c|c|c|c|}
\hline \multirow{2}{*}{$\begin{array}{c}\text { № } \\
\text { indicator }\end{array}$} & \multicolumn{3}{|c|}{ Astrakhanregion } & \multicolumn{3}{|c|}{ RepublicofAdygea } & \multicolumn{3}{|c|}{ RepublicofKalmykia } \\
\hline & Meaning & $\mathrm{Xi}$ & di & $\begin{array}{c}\text { Meanin } \\
\mathrm{g}\end{array}$ & $\mathrm{Xi}$ & di & Meaning & $\mathrm{Xi}$ & di \\
\hline 1 & 420.9 & 3.6 & 0.25 & 99.4 & 3.5 & 0.21 & 66.5 & 3.5 & 0.21 \\
\hline 2 & 106.6 & 3.7 & 0.27 & 31.5 & 3.5 & 0.22 & 12.9 & 3.5 & 0.20 \\
\hline 3 & 173.4 & 3.6 & 0.26 & 95.1 & 3.6 & 0.23 & 21.3 & 3.5 & 0.21 \\
\hline 4 & 284 & 4.3 & 0.49 & 330.6 & 4.5 & 0.58 & 204.9 & 3.9 & 0.34 \\
\hline 5 & 1320 & 4.4 & 0.52 & 906 & 4.9 & 0.68 & 998 & 4.8 & 0.65 \\
\hline 6 & 11.6 & 4.3 & 0.51 & 10 & 4.1 & 0.44 & 11 & 4.3 & 0.48 \\
\hline 7 & 11.5 & 4.6 & 0.59 & 12.4 & 4.5 & 0.55 & 9.6 & 4.8 & 0.66 \\
\hline 8 & 610.4 & 5.0 & 0.71 & 682 & 4.7 & 0.63 & 701.9 & 4.7 & 0.61 \\
\hline 9 & 420 & 3.8 & 0.29 & 95.3 & 3.5 & 0.22 & 18 & 3.5 & 0.20 \\
\hline 10 & 66.02 & 5.4 & 0.79 & 20.1 & 5.4 & 0.80 & 13.4 & 5.4 & 0.80 \\
\hline 11 & 499.9 & 3.8 & 0.30 & 192.3 & 3.5 & 0.22 & 124.4 & 3.5 & 0.21 \\
\hline 12 & 1653 & 4.0 & 0.39 & 2232 & 4.2 & 0.46 & 2269 & 4.2 & 0.46 \\
\hline \begin{tabular}{|c} 
The value of \\
the integral \\
indicator of \\
the level of \\
developmen \\
$t$
\end{tabular} & & D & 0.41 & & & 0.39 & & & 0.37 \\
\hline
\end{tabular}

And, as a result, the solution of the above problems logically implies an increase in the pace of socio-economic development of the regions of the Southern Federal District, including the Rostov Region. The implementation of the methodology for calculating the integral indicator of the level of regional development, reflecting the identified trends in the development of the regions of the Southern Federal District for 2011 - 2018 (see Fig. 2). will make it possible to implement the procedure of normalization of the results, which, in turn, will be the basis for constructing a fuzzy model for assessing the level of interregional integration (based on the apparatus of fuzzy sets) in further investigations and development of an integrated system for monitoring the degree of integrational ties between the regions of the Southern Russia.

Factors such as the level of transport communication and communications were discussed in detail above. Let us analyze them in relation to the partner regions under study and determine their participation in interregional integration economic processes in the Southern Federal District (Table 3), using the method of calculating the integral indicator of the socio-economic level of the development of regions.

An analytical assessment of the calculated data presented in the tables allow us to deduce that each of the studied regions has conditions for economic integration with the Rostov Region. The highest values of $\mathrm{D}=0,646$ and $\mathrm{D}=0,767$ indicate the most favorable interregional relations with the Krasnodar Territory in terms of the factors «Level of transport communication» and «Level of communications development» respectively. However, a deeper and more complete analysis taking into account the totality of all the considered groups of factors, when assessing the level of interregional integration, is 
possible on the basis of logical inference from the theory of fuzzy sets, and the calculated data of the integral indicator of the development level of the region are the initial data for describing the input attributes of the problem of modeling the object domain «level of integration relations».

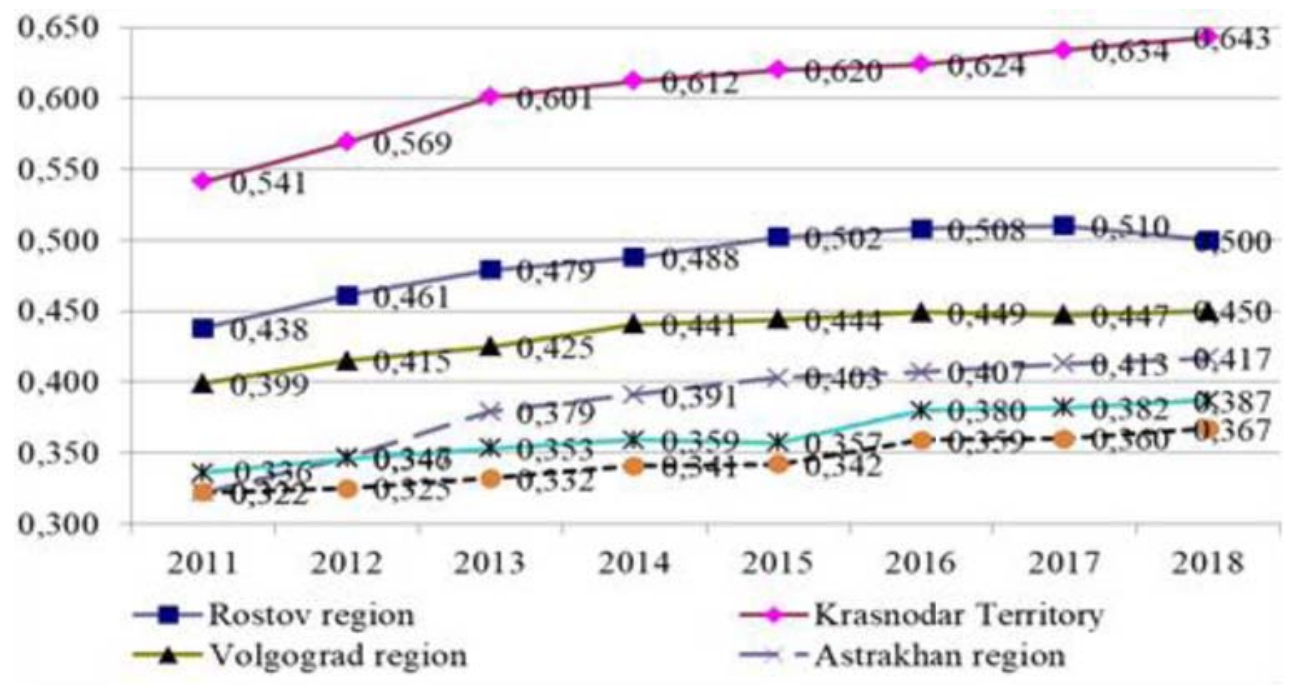

Fig. 2. Dynamics of the integral indicator of the socio-economic level of development of the regions of the Southern Federal District for $2011-2018$

After determining the trend of changes in the indicator of the development level of the regions of the Southern Federal District for 2011 - 2018 and performing the normalization procedure, the following stages of the analysis are: logical and linguistic description of input attributes of SEI, development of a knowledge base (based on fuzzy production rules), formation of a mechanism for fuzzy logical inference, development of a system for monitoring the level of integrative interaction of the regions of Southern Russia.

\section{Conclusion}

Acting as a driver-resource, interregional economic integration is a factor of economic development. The affordability of participation in the process of integration allows it to become an inexhaustible source of development of regional economies. It is an indispensable evolutionary, anthropogenic resource that all economic entities possess and that can be used for effective achievement of the state of integration of the trans-regional economic space.

It has been revealed that the dynamic distribution of resources, goods, and services under the conditions of interregional integration increases in case when the structure of the meta-region incorporates regions with different resource potential, in the interaction of which there appears a synergetic-cumulative effect of the impulse of economic development of the regions.

The scientific results of the study provide a conceptual basis for an in-depth analysis of the state of socio-economic processes in the regions of the Southern Russia, including the process of their interregional economic interaction as a form of integration process and a driver for the development of the single economic space of the Southern Federal District, 
improvement of efficiency of its functioning, and can be used by state regional management bodies (Ministries of Economic Development of the regions of the Southern Federal District and other Federal Districts of the Russian Federation) when developing strategies and programs to improve the mechanisms of interregional economic interaction, as well as research organizations involved in solving the problems of interregional integration.

The theoretical significance of the investigation results consists in the authors conceptual approach to the study of complex socio-economic systems and processes, including the process of interregional integration, functioning under the conditions of uncertainty and dynamically changing under the influence of many factors, the formalization and simulation of which have been implemented on the basis of the developed instrumental and mathematical apparatus, representing a system of the methodology of cognitive modeling of complex systems adapted to solving the problems of interregional economic integration and the apparatus of fuzzy sets for assessing the level and substantiating the directions of development of interregional integration, taking into consideration the qualitative nature of the factors that determine this level.

The conclusions and proposals obtained in this paper develop the methodology of analysis and predicting the behavior of complex economic systems and can act as a conceptual basis for justifying the directions of development and improving the efficiency of the processes of interregional economic integration.

\section{References}

1. L. Bozhko, Energy Procedia 147, 397-401, (2018) doi.org/10.1016/j.egypro.2018.07.109

2. M. Schröter, T. Koellner, R. Alkemade, S. Arnhold, et al, Ecosystem Services 31 (B), 231-241 (2018) https://doi.org/10.1016/j.ecoser.2018.02.003

3. E.N. Zakharova, E.E. Kardava, R.R. Avanesova, E.P. Avramenko, Regional Science Inquiry 8 (2), 45-54 (2016)

4. V.V. Prokhorova, E.N. Zakharova, Journal of Internet Banking and Commerce 21 (S4), 010 (2016)

5. N. Serbulova, et al., IOP Conf. Ser.: Earth Environ. Sci. 403, 012127 (2019) doi.org/10.1088/1755-1315/403/1/012127

6. N. Serbulova, et al., E3S Web Conf. 210, 02005 (2020) doi.org/10.1051/e3sconf/202021002005

7. A. Alukhanyan, K. Barmuta, O. Panfilova, D. Borisova, IOP Conference Series: Earth and Environmental Science, 403(1), 012044 (2019) doi.org/10.1088/17551315/403/1/012044

8. V. Kurdyukov, S. Kanurny, E3S Web of Conferences, 210, 13005 (2020) doi.org/10.1051/e3sconf/202021013005

9. T. Zhukova, O. Panfilova, I. Avlasenko, L. Avlasenko, E3S Web of Conferences, 175, 05028 (2020) doi.org/10.1051/e3sconf/202017505028

10. D.V. Borisova, G.V. Gorelova, International scientific journal «Stable development of mountain areas», 11 (1), 65-67 (2019)

11. D.V. Borisova, Cognitive modelling of interregional economic integration in terms of fuzzy data. Cognitive modelling: Proceedings of the Seventh International forum on cognitive modelling (5 - 15 September 2019, Retimno, Greece, i. Crete). In 3 parts.Part 
3. Cognitive modelling in science, culture, education: Proceedings of VII International conference «Cognitive modelling in science, culture, education. CMSCE-2019»Rostov-on-Don: Science and Education Fund, 243 - 252, (2019)

12. D.V. Borisova, G.V. Gorelova, N.D. Pankratova, Problems of interregional integration, cognitive modeling. 19th IFAC Conference on Technology, Culture and International Stability TECIS 2019Sozopol, Bulgaria, 26-28 September, 168-173, (2019) doi.org/10.1016/j.ifacol.2019.12.467

13. Y. Jiang, Trade, Foreign Direct Investment, and Development Strategies Chandos Asian Studies Series, 75-98, (2014) doi.org/10.1533/9781780634432.75

14. A. Landysh, T. Gadelshina, M. Vakhitova, Procedia Economics and Finance 24, 246250 (2015) doi.org/10.1016/S2212-5671(15)00655-3

15. T.F. Remington, Communist and Post-Communist Studies 48(1), 1-13 (2015) doi.org/10.1016/j.postcomstud.2015.01.005 